\title{
The Treatment of Essential Hypertension Based on Health Education
}

\author{
Fei Xie \\ Department of Nursing, School of Medicine, Yangtze University, Jingzhou, China \\ Email: xf1205@126.com
}

How to cite this paper: Xie, F. (2018) The Treatment of Essential Hypertension Based on Health Education. Yangtze Medicine, 2 262-270.

https://doi.org/10.4236/ym.2018.24028

Received: June 10, 2018

Accepted: December 18, 2018

Published: December 21, 2018

Copyright $\odot 2018$ by author and Scientific Research Publishing Inc. This work is licensed under the Creative Commons Attribution International License (CC BY 4.0)

http://creativecommons.org/licenses/by/4.0/

\section{(c) (i) Open Access}

\begin{abstract}
Objective: To investigate the treatment effect of essential hypertension based on health education. Methods: A randomized controlled field intervention trial was used in this study, patients with essential hypertension treated in community health services were subjects investigated, and psychological intervention should be carried out based on pharmaceutical services, to evaluate the effect of psychological intervention model based on pharmaceutical care on blood pressure control and overall health improvement in patients with essential hypertension. Results: Before treatment, systolic and diastolic blood pressure had no significant difference between two groups, P $>0.05$. After treatment, SBP and DBP in two groups were significantly decreased compared to that before treatment in the same group. After treatment, SBP and DBP in the intervention group were significantly decreased compared with normal group, $\mathrm{P}<0.05$. Before treatment, there was no significant difference in SRHMS scores of physical, psychological and social health between two groups $(P>0.05)$. After treatment, the scores of the physiological health subscale and the total scale of the control group, the scores of each subscale and the total amount of the intervention group were significantly higher than those before the same group, the difference was statistically significant $(\mathrm{P}<$ 0.05), and the scores of the mental, social health subscales and the total scale of the intervention group were significantly higher than those in the control group, and the differences were all statistically significant $(\mathrm{P}<0.05)$. Conclusion: Under the background of the new round of medical and health system reforms, this model will help health workers and community residents establish new types of doctor-patient relationship and improve the quality of life of chronic diseases such as hypertension.
\end{abstract}

\section{Keywords}

The Essential Hypertension, Health Education, Community, Psychological, Intervention 


\section{Introduction}

In recent years, the prevalence of hypertension in China is on the rise. The latest survey of nutritional and chronic conditions in the population showed that the prevalence of hypertension in adults who aged 18 years and above in China in 2012 was $25.2 \%$, which was significantly higher than that in 2002 . The etiology of hypertension is complicated, the course of disease is prolonged, the cure rate is low, and there are many complications. Patients and their families have been subjected to tremendous psychological and economic pressure. Psychological stress has become one of the risk factors to human essential hypertension. In most patients hypertension is often accompanied with anxiety, depression and other psychological disorders, which seriously affect their quality of life. Related studies have shown that the application of psychological intervention to the prevention and treatment of hypertension can avoid the inducement of bad society and psychological factors to the patients, reducing the incidence and mortality of hypertension. Pharmaceutical services provide drug-related services to the public with pharmaceutical expertise and tools, and the goal is to get positive results that improve the quality of patient's life [1]. As a main component of chronic non-communicable diseases in communities, the treatment of hypertension is a long-term process and needs drug combination. Practice in developed countries has proved that pharmaceutical care provided by community pharmacists can alleviate adverse drug reactions caused by long-term medication, reduce medical costs, and ensure safe and effective use of drugs, thereby improving the quality of life of patients [2]. However, the combination of psychological intervention and pharmaceutical care, was carried out on patients with essential hypertension in the community. This kind of practice activity is still rare in our country. Therefore, a randomized controlled field intervention trial was used in this study. Patients with essential hypertension treated in community health services were investigated and psychological intervention was carried out based on pharmaceutical services. The aim was to evaluate the effect of psychological intervention model based on pharmaceutical care on blood pressure control and overall health improvement in patients with essential hypertension.

\section{Materials and Methods}

\subsection{Research Object}

The investigation was approved by the Ethics Committee of Yangtze University. 100 patients with primary hypertension treated in this community were selected as the research object. For the research purposes, we set up inclusion and exclusion criteria, and inclusion criteria were: 1) According to the diagnostic criteria of WHO for hypertension; 2) the history of hypertension was more than 6 months, taking antihypertensive drugs or receiving antihypertensive treatment; 3) age between 50 and 80;4) voluntary participation in informed consent. Exclusion criteria: 1) severe visual and hearing impairment; 2) complicated complications (such as Grade III and above in heart failure, severe renal failure, malig- 
nant tumor, etc.); 3) previous or present mental and mental disorders, with severe mental and cognitive impairment, and being involved in other intervention research experiments. The patients were randomly divided into the control group and the intervention group, with 50 cases in each group. There were 50 cases in the control group, including 24 male patients and 26 female patients, aged between 51 and 78 , the average age was $(65.5 \pm 5.2)$ years, and the range of course was 1 - 3 years. There were 50 cases in the intervention group, including 22 male patients and 28 female patients. Aged between 50 and 77, the average age was $(64.8 \pm 5.4)$ years, and the range of course was $1-3$ years. There were no other malignant tumors in two groups, and their mental state was normal. There was no significant difference in clinical data between the two groups $(\mathrm{P}>0.05)$, which was comparable. All patients were suffered antihypertensive medication following the doctor's advice.

\subsection{Intervention Methods}

According to the related standards and regulations in "Guidelines for the management of hypertension in China" (revised in 2014), the control group was given routine antihypertensive drug therapy (dihydropyridine calcium antagonists) and routine management, while the intervention group was given pharmaceutical services (including hypertensive medication) and psychological intervention on this basis for 6 months (October 2013-March 2014). The team consisted of four community pharmacists and four psychological consultants, and it was divided into four groups, which had one community pharmacist and one psychological consultant. Our team trained community pharmacists and psychologists for two weeks, formulated the uniform pharmaceutical and psychological interventions to ensure each group intervention consistency with the content, step, and frequency. The intervention process was strictly controlled. Pharmaceutical services and psychological interventions were as follows:

1) Rational use of education was conducted by community pharmacists on regular health knowledge and drug therapy lectures. It was commonly introduced as basic information of used drugs, including the expected effect of drug treatment, adverse drug reaction and emergency treatment methods for patients. Files about drug use and health were established for each patient. Patients were supplied with regular door-to-door service, solving the problem for use, drug interactions and adverse drug reaction monitoring, timely correcting the wrong drug habit, increasing medication compliance, eliminating the unreasonable drug use factors. The patients were intervened 1 time every 2 weeks, and 1 hour per time. The total intervene is 12 times.

2) Health education was carried out by community pharmacists in the health education room, including: a) Giving out propaganda materials and the knowledge of hypertension lectures. Let patients understand the relationship between bad habits (smoking, drinking, high salt, high fat, high cholesterol diet, etc.) and cardiovascular disease, and motivate them to quit smoking, eat properly and exercise moderately. b) Strengthen successful experience. Patients were encouraged 
to recall and share the experience of correcting unhealthy life habits and their influence on hypertension control, and to strengthen patients' willingness to quit smoking and limit alcohol in a timely. c) Enhance the influence of model and enhance its confidence. d) Set goals and formulate action plans. Intervene 1 time every 2 weeks, 2 hours per time. The total intervene is 12 times.

3) Psychological intervention of cognitive behavior by psychological counselors, namely cognitive behavioral therapy (CBT). Through oneself and self-awareness to identify and understand the patient's mental state, and according to the result of their psychological questionnaires, using evaluation and challenge the idea, evaluate assumptions and rules, promote and transfer stimulus control, behavioral skills training and the habit of twisting therapy method, towards key issues (how to control and adjust negative emotions, how to enhance social adaptability, etc.) to carry out targeted treatment. The intervention was carried out in group mode, with 15 people in each group, and 10 to 15 times in 6 months, $1.5 \mathrm{~h}$ per time.

4) Music and relaxation therapy were selected for soothing. Beautiful music was played for 20 minutes before rational use, healthy education and psychological intervention. Guided by community pharmacists and psychologists, patients learned to relax scientifically and maintain peace of mind, to reduce the excitability of sympathetic nerve, alleviate the tension of blood vessel wall. This was carried out by group or team, group intervention not more than 60 people, team intervention not more than 20 people. This intervention can be used during lectures, group training. Patients can also carry out their own, unlimited number of times.

5) Social support is conducted by means of household visits, exchange of experience, telephone follow-up etc. including: a) Family support by encouraging family members to participate in the treatment of hypertensive patients, encouraging their active care, encouraging patients, and monitoring their medication as well as blood pressure control. b) Peer support. Using community health service center as a place to promote communication between patients and patients to share experiences in the prevention and treatment of hypertension and encourage and learn from each other. c) Community support: Post information on the prevention and treatment of hypertension in community health service center and neighborhood committees, 1 theme per issue, 1 for every 2 weeks. Open contact information of community pharmacists to facilitate timely communication between patients. Carry out telephone follow-up to guide patients with medication, and follow up on their blood pressure control. Social support run through the whole process of the study and was jointly completed by community pharmacists and psychological consultants.

\subsection{Blood Pressure Measuring Tools}

Blood pressure was measured by a community pharmacist with a standard mercury sphygmomanometer for the patient's right arm sitting blood pressure, 3 
times a week.

\subsubsection{Evaluation of Medication Compliance}

The compliance of patients was directly related to the development and therapeutic effect of hypertension [3]. The modified Morisky questionnaire [4] was used to evaluate the medication compliance of two groups. The investigation included: 1) Have you ever forgotten to take medication? 2) Do you sometimes do not pay attention to take medicine? 3 ) Do you stop the medicine when you feel your condition is improving? 4) Do you stop the medicine when you feel your condition is worsening? The above 4 questions were answered by "yes" or "no". If the 4 questions were all "no", then the medication compliance was "good". If one or more of them were "yes", then the medication compliance was "bad". The medication compliance evaluation was conducted 1 month after the intervention began and ended.

\subsubsection{The Evaluation of Knowledge of Drug Use}

Evaluating the knowledge of hypertension drug use in patients in two groups by self-made questionnaire. 10 questions were set in the questionnaire, including the commonly used hypertension drugs' name, usage and dosage, medication time, treatment goals, drug preservation methods, main adverse reactions and prevention, drug-food interactions, drug-drug interactions and complications. The patient answered 1 question correctly, with 1 point, and the full mark is 10 . 9 - 10 points are judged to be excellent, 6 - 8 are good, and less than 6 are bad. The knowledge of drug use was investigated before and after treatment.

\subsubsection{Evaluation of Health Status [5]}

The health status of hypertensive patients was evaluated by the self-assessment scale (SRHMS) [6]. The table consists of three evaluation forms, namely physiological, psychological and social health, there are 48 entries, the maximum value of each item is 10 , and the lowest value is 0 ; The theoretical maximum value of this table is $170,150,120$ and 440 , which is applicable to the health measurement of the population of 14 years or older. The score of SRHMS can directly reflect the health of the patient. The higher the score, the better the health is. SRHMS were distributed to both groups before and after treatment. After the patient truthfully fills in, the researcher receives on the spot.

\subsection{Statistical Methods}

The data were statistically analyzed by SPSS 17.0 software. The metering data was expressed as $\mathrm{x} \pm \mathrm{s}$, comparison between groups was conducted with independent sample t-test, in the group before and after treatment was conducted with paired t-test; the count data was expressed by the rate, and the comparison between the groups was conducted by $\mathrm{X}^{2}$ text. And SRHMS scores have a Wilcoxon test for statistical results. $\mathrm{P}<0.05$ was a statistically significant difference. 


\section{Results}

\subsection{The Comparison of Control and Intervention Groups on Antihypertensive Effect}

Before treatment, as regards the SBP and DBP there was no significant difference between the two groups, $\mathrm{P}>0.05$. After treatment, SBP and DBP in the two groups were significantly decreased compared with before treatment in the same group. After treatment, SBP and DBP in intervention group were significantly decreased compared with control group, $\mathrm{P}<0.05$. The detailed data showed in Table 1.

\subsection{The Comparison of SRHMS Scores in Control and Intervention Groups}

Before treatment, there was no significant difference in SRHMS scores of physical, psychological and social health between two groups $(P>0.05)$. After treatment, the scores of the physiological health subscale and the total scale of the control group, the scores of each subscale and the total amount of the intervention group were significantly higher than those before in the same group, the difference was statistically significant $(P<0.05)$, and the scores of the mental, social health subscales and the total scale of the intervention group were significantly higher than those in the control group, and the differences were all statistically significant $(\mathrm{P}<0.05)$. The SRHMS scores of the two groups before and after treatment were compared in Table 2.

Table 1. Comparison of antihypertensive effect between groups.

\begin{tabular}{|c|c|c|c|c|c|}
\hline \multirow{2}{*}{ Groups } & \multirow{2}{*}{$\mathrm{n}$} & \multicolumn{2}{|c|}{$\mathrm{SBP} / \mathrm{mmHg}$} & \multicolumn{2}{|c|}{$\mathrm{DBP} / \mathrm{mmHg}$} \\
\hline & & before & after & before & after \\
\hline Control & 50 & $155.5 \pm 14.6$ & $137.2 \pm 12.5^{\star}$ & $98.3 \pm 8.3$ & $84.2 \pm 7.2^{\star}$ \\
\hline Intervention & 50 & $156.4 \pm 13.8$ & $130.2 \pm 10.4^{\star \#}$ & $98.0 \pm 7.99$ & $79.4 \pm 8.4^{\star \#}$ \\
\hline
\end{tabular}

Note: ${ }^{\star} \mathrm{P}<0.05$ vs. before, ${ }^{\#} \mathrm{P}<0.05$ vs. control group.

Table 2. Comparison of SRHMS scores between two groups before and after treatment.

\begin{tabular}{cccc}
\hline & & Control & Intervention \\
\hline Physical score & Before & $84.2 \pm 18.9$ & $100.3 \pm 21.5$ \\
Asychological score & After & $84.9 \pm 20.3^{*}$ & $111.5 \pm 18.6^{*}$ \\
& Before & $97.9 \pm 27.5$ & $95.6 \pm 31.2$ \\
Social health score & After & $98.5 \pm 29.7$ & $120.5 \pm 26.6^{* *}$ \\
& Aefore & $76.8 \pm 22.7$ & $77.3 \pm 25.0$ \\
Total score & Before & $78.4 \pm 27.3$ & $103.4 \pm 22.1^{* *}$ \\
& After & $278.4 \pm 57.6^{*}$ & $262.0 \pm 59.3$ \\
\hline
\end{tabular}

Note: ${ }^{\star} \mathrm{P}<0.05$ vs. before; ${ }^{\sharp} \mathrm{P}<0.05$ vs. control group. 


\section{Discussion}

Hypertension is the most common chronic disease, which seriously jeopardizes national health and the quality of patient's life. Providing pharmacological services for hypertensive patients as well as implementing psychological interventions can help them eliminate negative emotions, stabilize their mentality, build confidence to overcome disease, and prevent adverse emotions from harming their own health, thereby improving the effectiveness of drug treatment and quality of patient's life. However, at present, the prevention and treatment of hypertension in China rarely combines pharmacy services with psychological interventions, and most of the subjects studied are hospitalized patients in large hospitals, fewer in the community [7]. Therefore, this study uses randomized-controlled and on-site intervention trials, and takes pharmacological services performed by community pharmacists as a vehicle to provide psychological intervention for patients who have essential hypertension in the community and promote the rational use of drugs in hypertensive patients, as well as strengthen the antihypertensive effect, then improve the patient's health.

Pharmacy services can improve the patients' drug compliance and the level of drug knowledge. In this study, the pharmacy service team led by community pharmacists went deep into the community to conduct on-site consultations, and introduced drug knowledge to patients by organizing lectures on rational use drug and distributing promotional materials, and through the improved Morisky questionnaire and self-prepared questionnaires to assess the drug compliance and the level of drug knowledge. Before treatment, the quantity of better drug compliance in both groups patients had less than $30 \%$, more than $60 \%$ of patients had poor level of medical knowledge and their understanding only stayed on the basic knowledge, such as drug name, usage, medication time, treatment goals and so on. After 6 months of pharmacy services, the proportion of patients in the intervention group who adhered to medications was significantly higher, and more patients could become familiar with common adverse reactions of antihypertension drugs and preventive measures, drug-drug interactions, drug-foods interactions and so on, indicating that good community pharmacy services can effectively improve the patient's drug compliance and the level of drug knowledge.

The psychological intervention based on pharmacy services can enhance the antihypertensive effect of patients. Modern medicine believes that the influence of psychological factors goes through the entire process of the disease. Negative emotions such as depressed mood, mental depression can inhibit the normal function of the autoimmune system and reduce body resistance to disease. Studies have shown that psychological factors such as anxiety, which introduced into the brain as stress, can lead to mental stress, depression, or indecision, and then cause negative emotions such as anxiety. The negative emotions represented by anxiety and depression can increase the risk of developing hypertension, which is not conducive to the control of blood pressure. The results of this study 
showed that the antihypertensive effect of the intervention group was significantly better than that of the control group. The reason is possible that the control group only used conventional drugs, while the intervention group also increased the pharmacy services and psychological intervention, as well as reduced the effects of anxiety and depression on blood pressure, and these make patient smoothly depressurized. The results of this study are like those investigations [8], suggesting that the implementation of psychological interventions based on pharmaceutical care can enhance the antihypertensive effect of patients.

Psychological interventions based on pharmacy services can improve the psychological and social health of patients. Studies have shown that various psychological factors are closely related to the occurrence, development, and outcome of hypertension [9]. In this study, patients in the intervention group performed cognitive therapy, social support, and other interventions in addition to pharmaceutical care based on conventional drug therapy to improve the patient's psychological state, relieve their anxiety, and increase their confidence and ability in overcoming the disease. After 6 months of intervention, the scores of the physical health subscales and total tables of the control group were higher than those of the same group before the treatment; while the scores of the physical health subscale and total table increased in the intervention group, The score of the healthy subscale was also significantly higher than that of the same group before treatment, suggesting that the psychological intervention measures achieved the desired effect.

Implementing psychological intervention on the basis of pharmacy services enriches the connotation of community health service practice. In China, community health services usually do not include pharmacists providing face-to-face professional services to patients. This study not only introduced pharmacy services in the prevention and treatment of chronic diseases in the community. At the same time, it also incorporates the practice of psychological intervention. Four community pharmacists and four psychological counsellors work together to complete the intervention. All four pharmacists graduated from pharmaceutical or clinical pharmacy majoring in domestic medical colleges. They have received psychology-related subject education such as doctor-patient communication and pharmacy services during their undergraduate study. They have already worked in pharmacy practice positions at least five years, with certain communication skills and work experience; four psychologists have passed the national psychological counsellor's secondary exam, obtained corresponding certificates, and received basic medical and health knowledge training. During the intervention process, the division of pharmacists and psychologists was clear, and both used their own expertise to provide services for patients with hypertension. Through practice, it has been proved that the comprehensive intervention model of pharmaceutical services and psychological intervention has achieved good results, can effectively promote blood pressure control and health recovery of patients, increase the level of rational drug use, and improve the psychological state of patients, which is worthy of promotion and application in community 
health services. Under the background of the new round of medical and health system reforms, this model will help health workers and community residents establish new types of doctor-patient relationship and improve the quality of life of patients with chronic diseases such as hypertension.

\section{Conflicts of Interest}

The author declares no conflicts of interest regarding the publication of this paper.

\section{References}

[1] Geldsetzer, P., Manne-Goehler, J., Theilmann, M., Davies, J.I., Awasthi, A., Vollmer, S., Jaacks, L.M., Bärnighausen, T. and Atun, R. (2018) Diabetes and Hypertension in India: A Nationally Representative Study of 1.3 Million Adults. JAMA Internal Medicine, 178, 363-372. https://doi.org/10.1001/jamainternmed.2017.8094

[2] Tomaka, J., Thompson, S. and Palacios, R. (2006) The Relation of Social Isolation, Loneliness and Social Support to Disease Outcomes among the Elderly. Journal of Aging and Health, 18, 359. https://doi.org/10.1177/0898264305280993

[3] Abqrall-Barbry, G. and Consoli, S.M. (2006) Psychological Approaches in Hypertension Management. Presse Medicale, 35, 1088.

[4] Hepler, C.D. and Strand, L.M. (1990) Opportunities and Responsibilities in Pharmaceutical Care. American Journal of Hospital Pharmacy, 47, 533.

[5] Daniali, S.S., Eslami, A.A., Maracy, M.R., Shahabi, J. and Mostafavi-Darani, F. (2017) The Impact of Educational Intervention on Self-Care Behaviors in Overweight Hypertensive Women: A Randomized Control Trial. ARYA Atherosclerosis, 13, 20-28.

[6] Xu, J., Hu, M., Liu, S. and Wang, Y.M. (2001) Item Analysis of Revised Version of Self Rated Health Rating Scale (SRHMSV1.0). Modern Rehabilitation, No. 19, 58-59.

[7] Black, R.E., Taylor, C.E., Arole, S., Bang, A., Bhutta, Z.A., Chowdhury, A.M.R., Kirkwood, B.R., Kureshy, N., Lanata, C.F., Phillips, J.F., Taylor, M., Victora, C.G., Zhu, Z. and Perry, H.B. (2017) Comprehensive Review of the Evidence Regarding the Effectiveness of Community-Based Primary Health Care in Improving Maternal, Neonatal and Child Health: 8. Summary and Recommendations of the Expert Panel. Journal of Global Health, 7, Article ID: 010908.

https://doi.org/10.7189/jogh.07.010908

[8] Odusola, A.O., Stronks, K., Hendriks, M.E., Schultsz, C., Akande, T., Osibogun, A., van Weert, H. and Haafkens, J.A. (2016) Enablers and Barriers for Implementing High-Quality Hypertension Care in a Rural Primary Care Setting in Nigeria: Perspectives of Primary Care Staff and Health Insurance Managers. Glob Health Action, 9, Article ID: 29041. https://doi.org/10.3402/gha.v9.29041

[9] Huang, S., Chen, Y., Zhou, J. and Wang, J. (2014) Use of Family Member-Based Supervision in the Management of Patients with Hypertension in Rural China. $\mathrm{Pa}$ tient Preference and Adherence, 8, 1035-1042. 